\title{
Higher frequency of medication overuse headache in patients attended by neurologists in Lima, Peru
}

\author{
María Elena Novoa' ${ }^{1}$ (D) , Carlos A. Bordini ${ }^{2,3}$ \\ 1'Department of Neurovascular Diseases, National Institute of Neurological Sciences, Lima, Peru \\ ${ }^{2}$ Neurologic Unit, FACEF Medical School, Brazil \\ ${ }^{3}$ Faculdade de Medicina de Ribeirão Preto, Universidade de São Paulo, São Paulo, Brazil
}

$凶$

Address all correspondence to María E. Novoa. National Institute of Neurological Sciences, Jr. Ancash 1271 Lima-Perú, e-mail: maria.novoa.m@upch.pe

Edited by:

Marcelo Moraes Valença

Keywords:

Headaches

Medication overuse headache $(\mathrm{MOH})$

Chronic daily headaches

Neurologists

General practitioners

Headache Disorders

Secondary

\begin{abstract}
Background

The type of medical care received (self-medication and/or medical care provided by a general practitioner or a neurologist) may be associated with differences in the frequency of medication overuse headaches.

Method

This cross-sectional analytical study included 222 records of patients with chronic daily headaches seen at the National Institute of Neurological Sciences Outpatient Unit in Lima, Peru. A pre-designed questionnaire was used to assess and categorize patients with frequent and chronic headaches.

Results

Ninety-four patients ( $42.34 \%$ of those with chronic daily headaches) met the criteria for medication overuse headache. Of these, $19(28 \%)$ self-medicated, $22(36 \%)$ consulted with the general practitioner, and the highest proportion of subjects, $53(58 \%)$, consulted with a neurologist. On bivariate analysis, subjects who had received care from a general practitioner and self-medicated were $38 \%$ and $51 \%$ less likely to have $\mathrm{MOH}$ than the subjects who received medical care from the neurologist $(p=0.012 ; 95 \% \mathrm{Cl} 0.42-0.90$ and $\mathrm{p}=0.001 ; 95 \%$ $\mathrm{Cl} 0.32-0.74)$. On multivariate analysis adjusting by sociodemographic and clinical factors, the association remained significant in regards to self-medication, but became marginal $(p=0.055)$ in regard to being seen by a general practitioner.

\section{Conclusion}

In this study, the frequency of the headache due to overuse of medication was higher in patients attending a neurologist than those attending a general practitioner or self-medicated. This cross-sectional design cannot assess whether this reflects more severe cases looking for specialized care or more medication overuse headaches as a result of inappropriate management.
\end{abstract}




\section{Introduction}

Headache is a symptom associated with many medical conditions (ICHD-3), and migraine and tension-type headache (TTH) are the most prevalent types of headaches. When migraine or TTH are categorized as episodic migraine (EM) and episodic TTH (ETTH) if attacks occur less than 15 days a month. If attacks occur for 15 or more days per month, they are defined as chronic daily headaches $(C D H$; chronic migraine $[\mathrm{CM}]$ or chronic TTH [CTTH] $]^{1,2}$; making up both groups $\mathrm{CM}$ and $\mathrm{CTTH}$, the majority of patients with chronic daily headaches $\mathrm{CDH} .{ }^{3}$ Medication overuse headache $(\mathrm{MOH})$ is a $\mathrm{CDH}$, mostly underlying a pre-existing primary headache, and adding to this, the overuse of symptomatic medication ( 10 to 15 days/month, depending on the overused drug, equal to or greater than three months) for acute episodes. ${ }^{2}$ The prevalence of $\mathrm{MOH}$ is $1 \%$ to $2 \%$ in the general population, predominantly in women in industrialized countries. ${ }^{4}$ The global burden of disease lists $\mathrm{MOH}$ as the $18^{\text {th }}$ cause of disability. In addition to disability due to migraine ( $6^{\text {th }}$ cause of disability); together, they represent the $3^{\text {rd }}$ leading cause of disability in the world. 4,5

Currently, the global campaign to reduce the burden of headache (Lifting the Burden) is assessing known and potential risk factors associated with $\mathrm{MOH}$. Several studies have determined associations with polymorphic variants related to the susceptibility of conversion to $\mathrm{MOH}^{6}$, psychopathological comorbidities ${ }^{7}$, within these, post-traumatic stress events ${ }^{8}$, depression, anxiety and insomnia ${ }^{9}$, and other sociodemographic factors, such as low education, place of residence, limited medical contact and type of medical care received in the development of $\mathrm{MOH} .{ }^{10-13}$ Factors that, in addition to transforming EM and ETTH into CM and $\mathrm{CTTH}^{7 \cdot 9,14,15}$, induce patients to seek different types of medical attention and to overuse medication in an effort to obtain relief from their pain. ${ }^{16.19}$

There is no clear evidence regarding the association of $\mathrm{MOH}$ with the type of medical care received. A 2012 Swedish publication suggested that limited medical care, due to reduced neurological care in rural areas, leads to self-medication with over-the-counter medication, increasing the likelihood of $\mathrm{MOH}$ development. ${ }^{10}$ Factor similar to the reality of developing countries such as Peru, where specialists are concentrated in metropolitan areas. This study evaluated whether there is a relationship between $\mathrm{MOH}$ and the type of medical care received (selfmedication, general practitioner or neurologist) in new patients who attended a neurological referral center.

\section{Methods}

Clinical interview - In this cross-sectional analytical study, we selected all records with $\mathrm{CDH}$ from a previous study that evaluated 419 new patients with headaches in the outpatient clinic of the National Instituto Nacional de Ciencias Neurológicas (INCN), Lima, Peru (a reference tertiary center). In the previous study, the participants were interviewed and examined by an INCN headache specialist, after the application of a complete clinical and demographic questionnaire.

Questionnaire - A questionnaire allowed the diagnosis of episodic and chronic headaches according to ICHD-III-Beta version-2013 criteria. ${ }^{20}$ For the study, we included from the primary study, data records with the diagnosis of $D C H$, excluding records with missing data. The questions: (1) Do you usually take any medication to relieve headaches?, (2) How many times a month do you take any medication?, and (3) How long have you been using the drug? allowed to generate the diagnosis of $\mathrm{MOH}$; while the questions (1) Has a doctor ever treated your headache in your life? and (2) What specialty did the treating physician have? allowed to categorize the type of medical care received.

Ethical aspects - The primary study protocol and informed consent form, as well as this secondary data analysis, were approved by the research and ethics committee of the INCN (N0523-2018-CIEI-INCN).

Data processing - Analysis was performed using Stata 14 StataCorp, (Texas, United States). Descriptive statistics are provided with measures of central tendency, frequencies, percentages, means and standard deviations as appropriate. We consider significance at $\mathrm{p}<0.05$ with two-tailed test. We compared proportions using chisquare $\left(X^{2}\right)$ test, after analysis of assumptions, to test the alternative hypothesis, that lack of neurological attention is associated with $\mathrm{MOH}$. Simple and adjusted regressions were performed using generalized linear models (GLM), binomial family to measure prevalence ratios (PR) due to our cross-sectional design. Due to the non-convergence of the binomial GLM model, a robust Poisson generalized linear model (GLM) was also run.

\section{Results}

From the primary study consisting of 419 participants (April 2016) (Figure 1), we selected 222 data records with 
$\mathrm{CDH}$, the total population of this cross-sectional study.

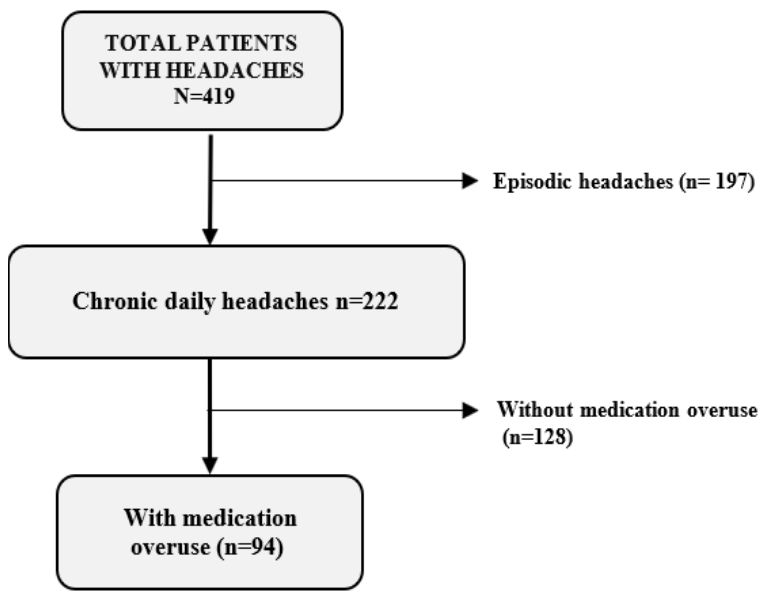

Figure 1. Flowchart for the screening of medication overuse headache patients. CEFAPERU data.

Sociodemographic and clinical variables are described in Table 1. The average age was $46( \pm 17$ SD) years. They were $146(66 \%)$ women and $76(36 \%)$ men. As in other studies, $\mathrm{CDH}$ is more prevalent in women; and more patients lived in urban areas, 124 (56\%). Migraines ( $n=122,55 \%)$ were the most frequent type of headache. The order of frequency of drug use was: drugs not remembered, $(n=83,38 \%)$, NSAIDs $(n=58,26 \%)$, paracetamol $(39,18 \%)$, other drugs $(24,11 \%)$ and ergotamine $(18,0.8 \%)$. Regarding the type of medical care received, neurological care was more frequent, occurring in 92 cases (41\%), followed by self-medication in 68 (31\%), and care by a general practitioner in 62 (28\%) (Figure 2).

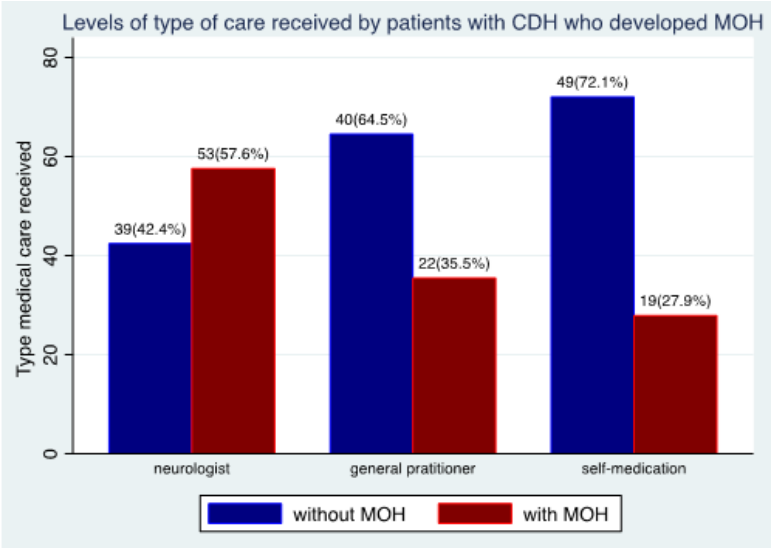

Figure 2. Levels of type of care received by patients with chronic daily headache who developed medication overuse headache $(\mathrm{MOH})$.

While all drug categories were overused; regression adjustment demonstrated that ergotamine were associated with a higher proportion of $\mathrm{MOH}$. Using neurological care as a category of reference in the simple regression, we demonstrated a $38 \%$ lower probability of $\mathrm{MOH}$ in patients seen by the general practitioner $(p=0.012,95 \% \mathrm{Cl} 0.42-0.90)$, and a $51 \%$ lower probability of developing $\mathrm{MOH}$ with self-medication ( $p=0.001,95 \% \mathrm{Cl} 0.32-0.74)$. Whereas, multiple regression adjusted for other sociodemographic and clinical variables, made the strength of association with general practitioner care marginal $(30 \%$ less probability, $p=0.053,95 \% \mathrm{Cl} 0.48$ $1.00)$, maintaining significant for self-medication $(37 \%$ less probability, $\mathrm{p}=0.030,95 \% \mathrm{Cl}$ 0.42-0.96) (Table 1).

\section{Discussion}

In this study, $\mathrm{MOH}$ was more frequent in patients seen by neurologists, compared to subjects seen by the general practitioner or self-medicated. In our view, the most plausible explanation is that individuals with $\mathrm{MOH}$ sought specialized care, although we cannot rule out that the higher frequency of $\mathrm{MOH}$ resulted from inadequate neurological management. Jonsson et al. ${ }^{10}$, in Sweden, reported that the high proportion of $\mathrm{MOH}$ in their study was due to limited access to medical care. However, in this series the frequency of $\mathrm{MOH}$ among those who received medical attention was non-significantly higher with neurological care than with care performed by other physicians. Longitudinal studies are required to confirm this hypothesis.

Most studies report that migraine is the most common underlying headache in $\mathrm{MOH}$, followed by $\mathrm{TTH}$. $^{18,21,22}$ Our data have this trend, although there was no significant difference. On the other hand, although psychiatric comorbidities such as anxiety, depression, and others, plus altered hormone levels associated with mood swings in women over 40 years of age ${ }^{7,23}$, could lead to $C D H$ and $M O H$, we found no significant differences for $\mathrm{MOH}$ in this subgroup.

Shand et al. ${ }^{11}$ reported that the overused drugs that led to the development of $\mathrm{MOH}$ in Argentina and Chile were combined ergotamine (70\%) and NSAIDs (33.8\%). Johnson et al. ${ }^{10}$, however, showed a higher proportion of $\mathrm{MOH}(66 \%)$ with overuse of psychotropic drugs. While Find et al. ${ }^{12}$, in a multicenter study on $\mathrm{MOH}$, reported that ergotamine was greatly overused in Latin America ( $72 \%$ compared with $4 \%$ in Europe, $p<0.001$ ). Our findings support these data, $\mathrm{MOH}$ was 2.5 times more frequent in patients with ergotamine overuse compared to those who overused drugs that they did not remember (reference category). In Peru, as in other Latin America countries ergotamine is extremely cheap and available over the counter.

Our study has several limitations. Self-report could create inaccuracies in the estimation of the exposure to the studied factors, affecting the reliability. However, a study on the validity of the self-report on $\mathrm{MOH}$ dependency severity 
Table 1. Analysis of the association between medication overuse headache and $(\mathrm{MOH})$ sociodemographic and clinical characteristics.

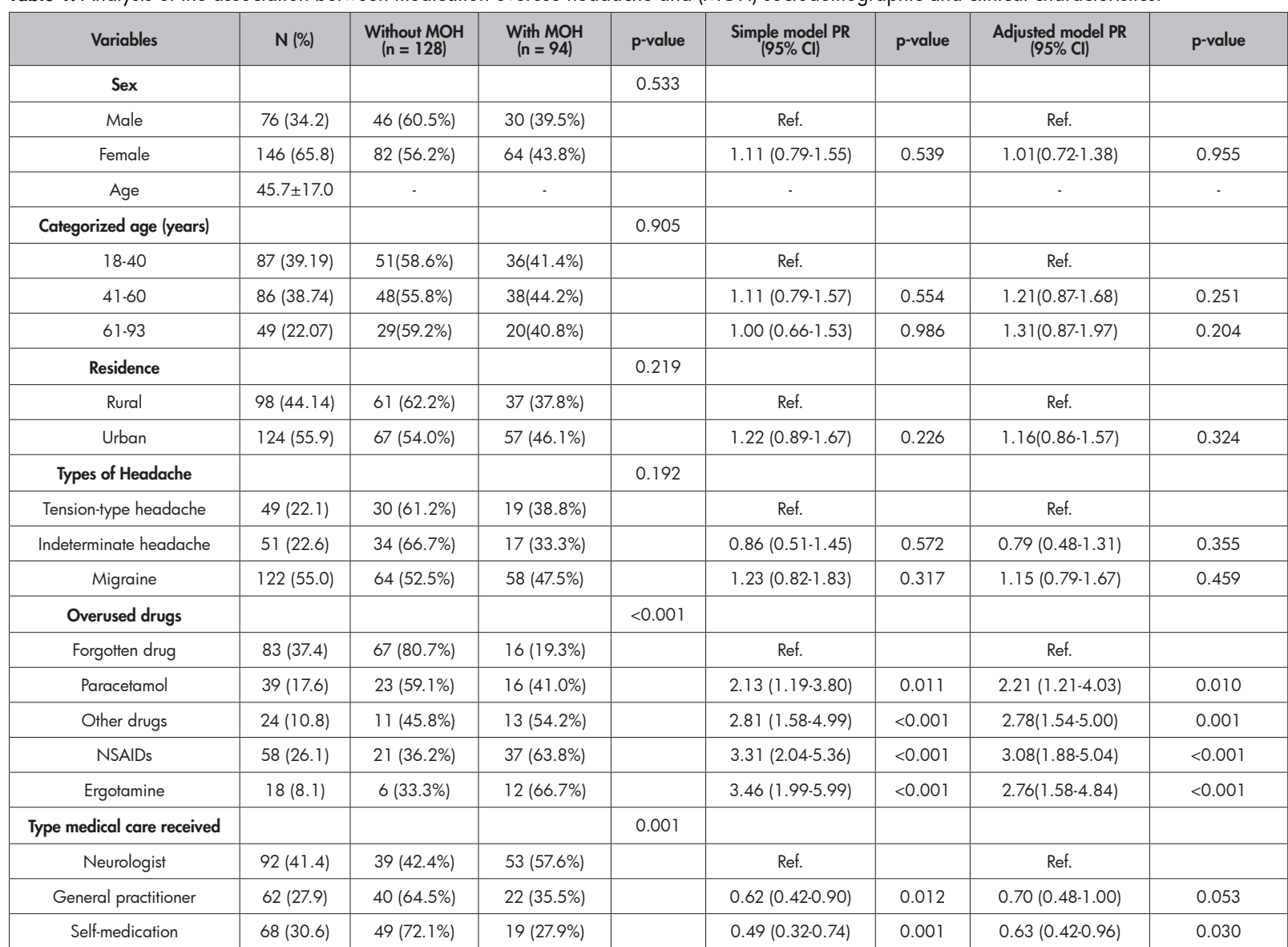

scales suggests that a self-reported version provides valuable information as a screening tool after a headache consultation. ${ }^{23}$ There are studies that provide greater security in this context, reporting concordance between what is reported by patients and medical prescription and diagnosis records, using telephone lines or online platforms for interviews. The crosssectional design of this study limits the estimation of causality, as well as the fact that this study was carried out in a tertiary referral center, where the most serious cases arrive, limiting the representativeness of $\mathrm{MOH}$ in the population.

We determined that subjects who arrived at this third-level center, with a higher proportion of $\mathrm{MOH}$, had already been seen by neurologists. These results could reflect that headaches as severe as $\mathrm{MOH}$, came in search of more specialized care than they received and are the result of improper prior, nonspecialized management. However, we cannot prove this from our data; longitudinal studies are required to clarify this and complement our findings. However, the high frequency of occurrence of $\mathrm{MOH}$ attended by neurologists, should lead to awareness regarding its diagnosis and management, as well as the prompt and adequate training of specialists in its diagnosis and management.

Conflict of interest: None

Author Contributions: MEN, Study conception and design, Data acquisition and interpretation, Revised the manuscript and final approval; $C A B$, Critically revised the manuscript and final approval.

María Elena Novoa

https://orcid.org/0000-0002-7545-9217

Carlos A. Bordini

https://orcid.org/0000-0002-1249-5202

\section{References}

1. Toom K, Braschinsky M, Obermann M and Katsarava Z. Secondary headache attributed to exposure to or overuse of a substance. Cephalalgia 2021;41(4):443. 452 Doi: $10.1177 / 0333102420942238$

2. Headache Classification Committee of the International Headache Society (IHS) The 
International Classification of Headache Disorders, 3rd edition. Cephalalgia 2018;38(1):1-211 Doi: $10.1177 / 0333102417738202$

3. Goadsby PJ and Boes C. Chronic daily headache. J Neurol Neurosurg Psychiatry 2002;72 Suppl 2(Suppl 2):ii2-ii5 Doi:10.1136/innp.72.suppl_2.ii2

4. Russell MB. Epidemiology and management of medication-overuse headache in the general population. Neurol Sci 2019;40(Suppl 1):23-26 Doi:10.1007/s10072-019-03812-8

5. Global, regional, and national incidence, prevalence, and years lived with disability for 354 diseases and injuries for 195 countries and territories, 1990-2017: a systematic analysis for the Global Burden of Disease Study 2017. Lancet 2018;392(10159):1789-1858 Doi:10.1016/s0140-6736(18)32279-7

6. Cargnin S, Viana M, Sances G, Tassorelli $C$ and Terrazzino $S$. A systematic review and critical appraisal of gene polymorphism association studies in medicationoveruse headache. Cephalalgia 2018;38(7):13611373 Doi: $10.1177 / 0333102417728244$

7. Migliore S, Paolucci M, Quintiliani L, Altamura C, Maffi $S$, D'Aurizio G, . . . Vernieri F. Psychopathological Comorbidities and Clinical Variables in Patients With Medication Overuse Headache. Front Hum Neurosci 2020; 14:571035 Doi:10.3389/fnhum.2020.571035

8. Bottiroli S, Galli F, Viana M, Sances G and Tassorelli C. Traumatic Experiences, Stressful Events, and Alexithymia in Chronic Migraine With Medication Overuse. Front Psychol 2018;9:704 Doi:10.3389/ fpsyg.2018.00704

9. Pompili M, Innamorati M, Lamis DA, Serafini G, Ricci F, Migliorati $M, \ldots$ Martelletti P. Depression and insomnia are independently associated with satisfaction and enjoyment of life in medication-overuse headache patients. Int J Psychiatry Med 2016;51(5):442-455 Doi:10.1177/0091217416680804

10. Jonsson $P$, Linde $M$, Hensing $G$ and Hedenrud $T$. Sociodemographic differences in medication use, health-care contacts and sickness absence among individuals with medication-overuse headache. J Headache Pain 2012;13(4):281-290 Doi:10.1007/ s10194-012-0432-y

11. Shand B, Goicochea MT, Valenzuela R, Fadic R, Jensen R, Tassorelli $C$ and Nappi G. Clinical and Demographical Characteristics of Patients with Medication Overuse Headache in Argentina and Chile: Analysis of the Latin American Section of COMOESTAS Project. J Headache Pain 2015; 16:83 Doi:10.1186/ s10194-015-0561-1

12. Find NL, Terlizzi R, Munksgaard SB, Bendtsen L, Tassorelli C, Nappi G, ... J Jensen R. Medication overuse headache in Europe and Latin America: general demographic and clinical characteristics, referral pathways and national distribution of painkillers in a descriptive, multinational, multicenter study. J Headache Pain 2015;17:20 Doi:10.1186/ s10194-016-0612-2

13. Viana M, De Icco R, Allena M, Sances G, Højland JR, Katsarava Z, . . . Tassorelli C. Clinical Subtypes of Medication Overuse Headache - Findings From a Large Cohort. Headache 2019;59(9):1481-1491 Doi: 10.1111 /head. 13641

14. Chen PK and Wang SJ. Medication Overuse and Medication Overuse Headache: Risk Factors, Comorbidities, Associated Burdens and Nonpharmacologic and Pharmacologic Treatment Approaches. Curr Pain Headache Rep 2019;23(8):60 Doi:10.1007/s 1 1916-019-0796-7

15. Mohseni N, Togha M, Arzaghi SM, Nekooie S, Tafti MF and Fatehi F. Personality Traits and Anxiety and Depressive Disorders in Patients With MedicationOveruse Headache Versus Episodic Migraine. Prim Care Companion CNS Disord 2017;19(6):17m02188 Doi:10.4088/PCC. $17 \mathrm{m02188}$

16. Peck KR, Roland MM and Smitherman TA. Factors Associated With Medication-Overuse Headache in Patients Seeking Treatment for Primary Headache. Headache 2018;58(5):648-660 Doi:10.1111/ head. 13294

17. Lundqvist C, Gossop M, Russell MB, Straand $J$ and Kristoffersen ES. Severity of Analgesic Dependence and Medication-overuse Headache. I Addict Med 2019;13(5):346-353 Doi:10.1097/ adm.0000000000000504

18. Jay GW and Barkin RL. Primary Headache DisordersPart 2: Tension-type headache and medication overuse headache. Dis Mon 2017;63(12):342-367 Doi:10.1016/i.disamonth.2017.05.001

19. Guo S, Shalchian S, Gérard P, Küper M, Katsarava $Z$, Ashina $M$ and Schoenen J. Prevalence of right-to-left shunts on transcranial Doppler in chronic migraine and medication-overuse headache. Cephalalgia 2014;34(1):37-41 Doi: $10.1177 / 0333102413497600$

20. The International Classification of Headache Disorders, 3rdedition (betaversion). Cephalalgia 2013;33(9):629808 Doi:10.1177/0333102413485658

21. López Martínez MDV, Pareja Román J, Jiménez Hernández MD, Maestu Unturbe $C$ and RamírezCastillejo MDC. Chronic Migraine with Medication Overuse: Clinical Pattern and Evolution from a Retrospective Cohort in Seville, Spain. SN Compr Clin Med 2020;2(9):1514-1525 Doi:10.1007/s42399. 


\section{0-00424-8}

22. Viana M, Bottiroli S, Sances G, Ghiotto N, Allena M, Guaschino $E_{1}$. . . Tassorelli $C$. Factors associated to chronic migraine with medication overuse: A crosssectional study. Cephalalgia 2018;38(14):2045-2057 Doi: $10.1177 / 0333102418761047$

23. Kristoffersen ES, Benth J, Straand J, Russell MB and Lundqvist $C$. Validity of self-reported assessment of
Severity of Dependence Scale in Medication-Overuse Headache. Scand J Pain 2019;19(4):837-841 Doi:10.1515/sjpain-2019-0022

24. Eichler GS, Cochin E, Han J, Hu S, Vaughan TE, Wicks $P, \ldots$ Devenport J. Exploring Concordance of PatientReported Information on PatientsLikeMe and Medical Claims Data at the Patient Level. J Med Internet Res 2016; 18(5):e1 10 Doi:10.2196/jmir.5130 